\title{
Decreased fetal movement: Is it an alarm to Obstetrician and Pregnant Lady?
}

\section{Padma Raj Dhungana $^{1}$, Rajesh Adhikari ${ }^{1}$, Prem Raj Pageni ${ }^{2}$, Apsara Koirala ${ }^{3}$}

${ }^{1}$ Department of Gynecology and Obstetrics, Pokhara Academy of Health Sciences, Western Regional Hospital

${ }^{2}$ Matrisishu Miteri Hospital, Batulechaur, Pokhara, Nepal

${ }^{3}$ Nobel Academy, Department of Pharmacology, Pokhara, Nepal

\section{Correspondence:}

Dr. Padma Raj Dhungana

Department of Obstetrics and Gynecology

Pokhara Academy of Health Sciences

Western Regional Hospital, Pokhara, Nepal

Article recived : 17th July 2018

Article accepted : 2nd Aug. 2018

\begin{abstract}
Introduction:

Fetal activity serves as an indirect measure of the fetus central nervous system integrity and function. Regular fetal movement can be regarded as an expression of fetal wellbeing. Maternal perception of decreased fetal movement is associated with poor feto-maternal outcome.

This study is to find out feto-maternal outcome in cases of decreased fetal movement, done by assessing fetal heart rate (FHR) correlating with cardiotocography (CTG) and USG (ultrasonography) findings, Apgar score and meconium in amniotic fluid.

Materials and method

This was a hospital based, prospective comparative study done in Patan Academy of Health Sciences, Lalitpur, Nepal. The sample size was 200; 100 cases of decreased fetal movement versus 100 cases of good fetal movement at same gestational age in weeks. Data processing was done in SPSS version 20.0 (SPSS, Chicago, IL, USA) and calculated by chisquare test. $P$ value less than 0.05 was considered significant.

Results:

Significant difference was found in fetal kick chart counting. In the decreased fetal movement group, $16 \%$ had oligohydraminos, $3 \%$ had polyhydraminos and $5 \%$ had non reassuring CTG and $4 \%$ had abnormal heart rate detected clinically. There was statistically significant difference observed btween the two groups in labor induction, cesarean section, meconium stain liquor, cord around the neck and different neonatal morbidities.

Conclusion:

Decreased fetal movement is associated with poor fetomaternal outcome. Improved vigilance, early identification, further evaluation with CTG, USG and proper management of these pregnancies have favourable pregnancy outcome.

Key words: Decreased fetal movement, feto-maternal outcome
\end{abstract}

\section{INTRODUCTION}

Fetal movement indicates that the fetus is alive. The integrity and function of central nervous system of fetus is indirectly measured by fetal movement. Regular fetal movement can therefore be regarded as an expression of fetal well-being. As pregnancy advances there is gradual decline in number of fetal movements (result of improved co-ordination) and reduced amniotic fluid volume coupled with the increased fetal size. ${ }^{1}$

The fetus conserves energy in chronic hypoxic condition. There is reduction in fetal movements as an adaptive mechanism to reduce oxygen consumption. There are different definitions of decreased fetal movement. No fetal movement for at least 12 hours with audible fetal heart beats and 10 or less fetal 
Orginal Article Decreased fetal movement : Is it an alarm to Obstetrician and Pregnant lady Dhungana P R et al.

movements for 12 hours is an alarm to mother and treating obstetrician. ${ }^{2}$

Maternal perception of decreased fetal movement is a common reason for visit to the antenatal clinic or delivery room. Studies have shown that poor fetomaternal outcome is a consequence of reduction or cessation of fetal movements. Fetal movement pattern perceived by mother can depend on various factors like anxiety, busy schedule, alcohol, sedative or corticosteroids consumption, fetal sleep, IUGR, fetal hypoxia, hypothyroidism, fetal anemia, neuromuscular abnormality and poly or oligohydramnios. The fetus diminishes its activity to reduce oxygen consumption in hypoxic condition and is an expression of fetal distress and placental dysfunction. Thus, there is need for fetal assessment in this situation. New methods of fetal assessment in various clinical settings have been introduced which includes real time USG, NST (CTG) CST, vibroacoustic stimulation, Doppler velocimetry of umbilical artery and uterine artery and biophysical profile. $^{3}$

\section{MATERIALS AND METHODS}

This was a hospital based prospective comparative study conducted at Patan Academy of Health Sciences, Lalitpur, Nepal.

During the study period from 15th May 2011-15th November 2011, total no of deliveries were 4689, in average 700 deliveries per month and 25 deliveries per day. 334 cases complaining decreased fetal movement were admitted among 4689 cases.

Every pregnant woman with history of decreased fetal movement attending in admission room or already admitted in antenatal ward were examined daily till obtaining required sample size i.e. 100 cases of decreased fetal movements. A detail history was taken, gestational age was verified by LMP or early scan and detail general physical, systemic examination was done.

Hundred cases as control of the same gestational age in weeks but with adequate fetal movement were taken immediately in admission or antenatal ward after verifying the gestational age by LMP or early scan if present and those fulfilling inclusion criteria were enrolled in study after taking informed consent. They were followed till delivery as those of decreased fetal movement group and findings were noted in a similar way.

The study was conducted after ethical approval from
IRB, NAMS. Written informed consent was taken from the patients.

All pregnant women with decreased fetal movement at term with singleton cephalic presentation were included as cases in the study and pregnant women with multiple pregnancies, mal-presentation, previous cesarean section, hypertensive disorder, APH and chronic medical diseases like diabetes were excluded from the study.

After enrollment of each case of decreased fetal movement, initial FHR status was recorded as i) FHR110-160 beats per minute, ii) FHR less than 110 iii) FHR more than 160 beats per minute and iv) no FHR audible, whichever may be the finding. Those with normal heart rate by auscultation were advised to maintain fetal kick chart counting by giving a paper and were asked to tear on the margin of paper when she felt fetal movements in a given time period in left lateral position. 10 fetal movements in 12 hours i.e. Cardiff count of 10 was taken as standard. At the same time cases with decreased fetal movements were subjected to CTG and finding were recorded as reassuring or non-reassuring and USG findings were recorded as fetal movement in USG present or absent and liquor volume normal(AFI $8-25 \mathrm{~cm})$, oligohydramnios $(<8 \mathrm{~cm}$ ) or polyhydramnios $(>25 \mathrm{~cm}$ ). Cases with decreased fetal movement were followed up till delivery. Mode of delivery togther with indications if instrumental or caserean section were recorded. Fetal outcome in terms of alive or still birth (fresh or macerated), Apgar score at 5 minutes, liquor status clear or meconium stained and cord around the neck were recorded. From babies admitted in nursery notes were taken about different morbidities like birth asphyxia, LBW, large for gestational age, meconium aspiration syndrome together with the duration of stay in NICU. The cause of mortality, if any, was recorded. Data analysis was done by SPSS version 23. Chisquare test was carried out. $\mathrm{P}$ value was considered significant if it was $<0.05$.

\section{RESULT}

During the study period of 6 months (15th May 2011 to 15th November 2011), 7250 obstetrics cases were admitted and among them, cases of decreased fetal movement at term were 362 . The incidence of decreased fetal movement was $5 \%$ in this study. 
Orginal Article Medical Journal of Pokhara Academy of Health Sciences (MJPAHS) Vol. 1 Issue 2 Jul-Dec 2018

\begin{tabular}{|c|c|c|c|}
\hline $\begin{array}{l}\text { Condition of fetus } \\
\text { during initial evaluation }\end{array}$ & $\begin{array}{c}\text { Among decreased } \\
\text { fetal movements } \\
\text { No. }(\%)\end{array}$ & $\begin{array}{l}\text { Among good fetal } \\
\text { movements } \\
\text { No. ( } \%)\end{array}$ & $P$ value \\
\hline \multicolumn{4}{|l|}{ Normal heart rate } \\
\hline (110-160 /min regular) & $96(96.00)$ & $100(100)$ & 0.121 \\
\hline \multicolumn{4}{|l|}{ Abnormal heart rate } \\
\hline$(<110$ or $>160 / \mathrm{min})$ & $4(4.00)$ & $0(0.00)$ & \\
\hline \multicolumn{4}{|l|}{ No fetal heart sound } \\
\hline Total & 100 & 100 & \\
\hline
\end{tabular}

No significant difference in heart rate on initial evaluation among two groups ( $P$ value 0.121 ). Abnormal FHR among decreased fetal movement cases were $4 \%$ ( 1 case had fetal bradycardia and 3 cases had fetal tachycardia) while non among those with good fetal movements.

Table 2: Fetal status assessment by CTG among cases with decreased fetal movements.

$\begin{array}{lcc}\text { CTG findings } & \text { No. } & \% \\ \text { Reassuring } & 95 & 95.00 \\ \text { Non reassuring } & 5 & 5.00 \\ \text { Total } & 100 & 100.00\end{array}$

Fetal status was assessed in all cases of decreased fetal movements with CTG. 95\% had reassuring CTG while $5 \%$ had non-reassuring. Among 4 cases having abnormal FHR on initial evaluation, 1 case with fetal bradycardia had multiple deceleration and 3 cases with fetal tachycardia had persistent fetal tachycardia with repeat CTG. Fifth case had no variability even in repeated CTG.

Table 3: USG findings among cases with decreased fetal movements

\begin{tabular}{|c|c|c|c|}
\hline \multirow{2}{*}{$\begin{array}{l}\text { USG finding } \\
\text { Fetal movemen }\end{array}$} & & No. & $\%$ \\
\hline & & & \\
\hline \multirow[t]{2}{*}{ in USG } & Present & 99 & 99.00 \\
\hline & Absent & 1 & 1.00 \\
\hline Liquor volume & Normal $(8-25 \mathrm{~cm})$ & 81 & 81.00 \\
\hline \multicolumn{2}{|c|}{ Oligohydromnios $(<8 \mathrm{~cm})$} & 16 & 16.00 \\
\hline \multicolumn{2}{|c|}{ Polyhydramnios $(>25 \mathrm{~cm})$} & 3 & 3.00 \\
\hline
\end{tabular}

All cases of decreased fetal movements were evaluated with ultrasound to see fetal movement and liquor volume. Abnormal ultrasonographic findings were on $20 \%$ of cases. In 1 case, there was no fetal movement along with AFI 2 and FHR 100 beats per minute in USG which had late deceleration in CTG.
Three cases $(3 \%)$ had polyhydraminos and 16 cases $(16 \%)$ had oligohydraminos

Figure 1: Comparison between modes of deliveries in decreased fetal movements and good fetal movement cases.

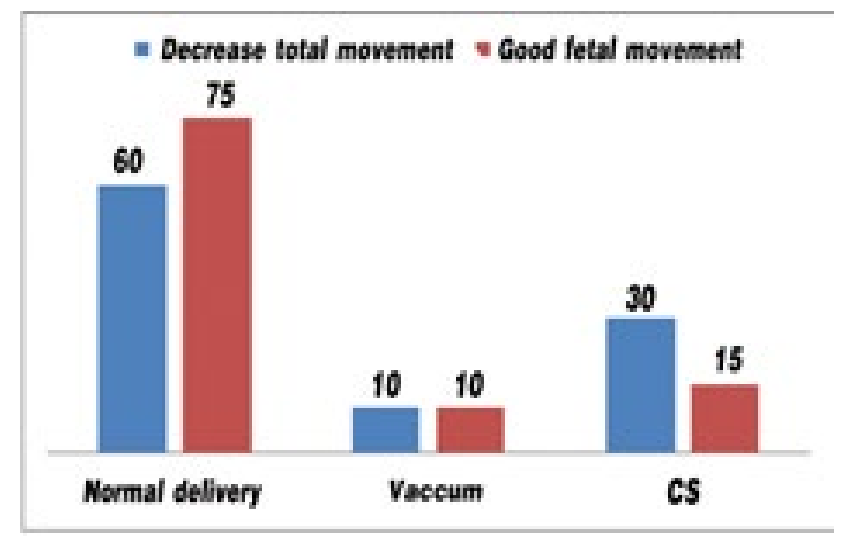

Figure shows statistically significant difference in cesarean section $30 \%$ versus $15 \%$ ( $P$ value 0.009 ) and normal delivery $60 \%$ versus $75 \%$ ( $P$ value 0.023 ) between decreased versus good fetal movement cases respectively. The instrumental deliveries (all vacuum deliveries) were $10 \%$ in both groups.

Figure 2.

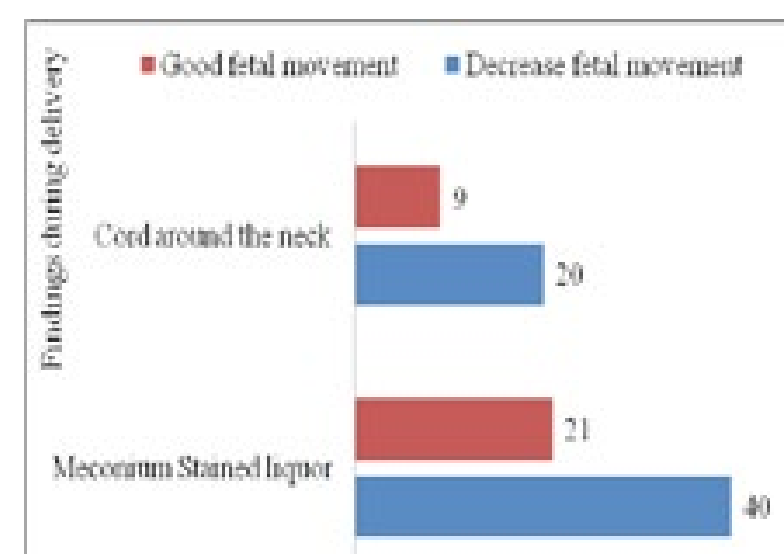

There was no significant difference in the findings like meconium stained liquor $40 \%$ versus $21 \%$ ( $P$ value 0.005 ) and cord around the neck $20 \%$ versus $9 \%$ $(P$ value 0.043 ) among decreased fetal movement versus good fetal movement respectively during the time of delivery. No congenital anomalies were detected in both groups.

There was no significant difference in apgar score between two groups. In apgar score at 5 minutes, 
Orginal Article Decreased fetal movement : Is it an alarm to Obstetrician and Pregnant lady Dhungana P R et al.

1 case had apgar score 3, 2 case had apgar score 5 and 97 cases had more than 7 among decreased fetal movement group while among those with good fetal movements all had apgar score more than 7 .

Figure 3: Fetal outcome in relation to Apgar score at 5 minutes.

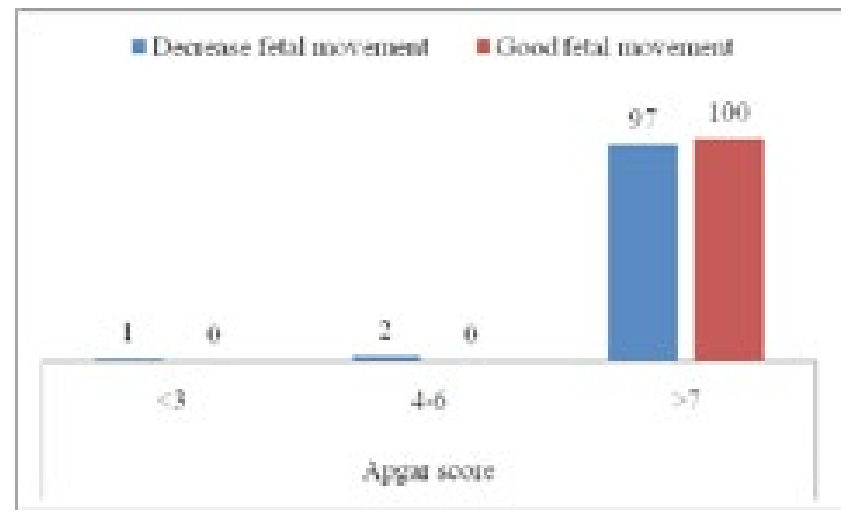

Table 4: Different neonatal morbidities admitted to Nursery.

$\begin{array}{lccc}\begin{array}{lcc}\text { Admission to } \\ \text { nursery for }\end{array} & \begin{array}{r}\text { Among decreased } \\ \text { fetal movements }\end{array} & \begin{array}{c}\text { Among good } \\ \text { fotal movements }\end{array} & \text { P value } \\ \text { Birth asphyxia } & 5(5 \%) & \begin{array}{c}\text { No. }(\%) \\ 0(0.00)\end{array} & 0.059 \\ \begin{array}{l}\text { Meconium aspiration } \\ \text { syndrome }\end{array} & 5(5 \%) & 0(0.00) & 0.059 \\ \text { Birth weight } & 6(6 \%) & 7(7 \% .00) & 1.000 \\ \text { Congenital anomalies } & 0(0.00) & 0(0.00) & \\ \text { Total } & 16(16 \%) & 7(7 \%) & \end{array}$

There was significant difference in neonatal morbidities requiring nursery admission $16 \%$ versus $7 \%(p=0.031)$ among decreased versus good fetal movement cases respectively. Decreased fetal movement group with neonatal morbidities like birth asphyxia and meconium aspiration syndrome had higher rate of nursery admission while good fetal movement group having abnormal birth weight $(<2$ and $>4 \mathrm{~kg}$ ) had higher rate of nursery admission. There was only one neonatal death in decreased fetal movement group.

\section{DISCUSSION}

Decreased fetal movement is a common condition to visit the antenatal clinic or admission room. In this study the incidence of decreased fetal movement was $5 \%$. In a study by Heazell AEP, Caroline MG Flenandy WV and Froen JF in 2008, it was 5-15\%. ${ }^{4}$

In our study, initial fetal condition evaluation was done by hearing FHR for one minute during admission time and half hourly after admission. Out of 100 cases of decreased fetal movement, four cases had abnormal FHR on initial evaluation. Among these, one had FHR 100 beats per minute and other three cases had FHR more than 160 beats per minute.

For assessment of fetal well-being and identification of the compromised fetus, antenatal fetal surveillance is necessary. Auscultation and CTG is the method frequently used to record the FHR. ${ }^{5}$ Antenatal fetal heart monitoring includes both non-stress tests (NST) and contraction stress tests (CST). FHR patterns are classified as either reassuring or non-reassuring depending on baseline heart rate, variability, and decelerations. ${ }^{6}$

Several studies have described the correlation of NST and CST with the subsequent perinatal outcome. In a randomized controlled trial, Flynn et al had shown a significant association of NST tracings with stillbirths, neonatal deaths, fetal growth restriction, complications of intrauterine hypoxia, and low Apgar scores. ${ }^{7}$ In a study by Rayburn et al, NST tracings within 1 week of delivery and subsequent perinatal outcome was studied where perinatal mortality in patients with reassurung NSTs was lower than untested patients or patients with non-assuring NSTs. ${ }^{8}$

Evertson et al assessed perinatal death within 1 week of negative CST where there were only 7 perinatal deaths in 680 patients. These were due to factors other than placental insufficiency, suggesting low rates of false negative CST. ${ }^{9}$

In our study, fetal status was assessed by cardiotopography among decreased fetal movement cases only. Among 100 cases of decreased fetal movement $95 \%$ had reassuring CTG. Ray burn et al, however, reported large proportion (56\%) of abnormal CTG traces in women perceiving decreased fetal movement which was predictive of an unfavorable perinatal outcome. ${ }^{10}$

In our study, $5 \%$ had non reassuring CTG. One case which had fetal heart sound 100 beats per minute showed late deceleration of significant amount and three cases with FHR more than 160 had persistent fetal tachycardia and one case had no variability on CTG and all underwent emergency cesarean section. In a study by Rabin Owitz et al adequate acceleration have been reported in the association with $79 \%$ of fetal movements perceived by the mother and $99 \%$ of fetal movements seen sonographically. ${ }^{11}$ In a study by Lee and Drukker, FHR decelerations in CTG for 1 
minute or longer were associated with an increased rate of cesarean delivery \& fetal demise. ${ }^{12}$ While Valentine and Marsal found that only few maternally perceived decreased fetal movement coincided with abnormal CTG traces and they underwent cesarean section with no perinatal mortality. ${ }^{13}$ Haezell AEP et al found USG as $91.3 \%$ specific, $33.3 \%$ sensitive with positive predictive value of $50 \%$ and negative predictive value of $73.3 \% .{ }^{14}$ Whitty et al. found $9 \%$ of patient had incidental abnormal ultrasonographic findings providing useful information in cases with indecreased fetal movement. ${ }^{15}$

In the present study, among 100 cases of decreased fetal movement only one case had no fetal movements on USG even on ultrasound examination. This cases had severe oligohydramnios, FHR 100 beats per minute with late deceleration in CTG and biophysical profile 2 with reversed diastolic flow on umbilical artery velocimetry. Remaining $99 \%$ had fetal movement on USG.

Amniotic fluid volume is an important determinant of fetal activity. Sherer et-al observed that restricted intra uterine space limits fetal movement as a result of decreased amniotic fluid and may be associated with fetal malformation. ${ }^{16}$ Locatelli et al. reported poor perinatal outcome among cases with oligohydramnios. ${ }^{17}$ Baron et al observed that women with low AFI were more likely to have Caesarean section for fetal distress than women with normal AFI, but the rates of neonatal complications were similar. ${ }^{18}$ In the present study, liquor volume study among 100 cases of decreased fetal movement in ultrasound showed $16 \%$ oligohydramnios, $3 \%$ polyhydramnios and $81 \%$ had normal AFI.

In present study there were $60 \%$ normal delivery, $30 \%$ cesarean delivery and $10 \%$ instrumental delivery (all vacuum delivery). While $75 \%$ normal delivery, $15 \%$ cesarean delivery and $10 \%$ vacuum delivery were among cases with good fetal movements. Meconium stain fluid was found in 40 cases with decreased fetal movement while only in 21 cases with good fetal movements. Cord around the neck was found in 20 neonates with decreased fetal movements and 9 neonates with good fetal movements during delivery. No congenital anomalies were found in both the groups which is comparable with the study done by Sinha $\mathrm{D}$ et al. where $32 \%$ women in study group needed intervention due to fetal compromise campared to $21 \%$ in control group. Overall cesarean section rate was $24 \%$ in study group and $14 \%$ in control group. In study group there were $4 \%$ fetal distress in antepartum period and $28 \%$ during labor compared to no fetal distress in antepartum period and $21 \%$ during labor in control group. ${ }^{19}$

In our study, baby with apgar score at 5 minutes less than 3 was 1, apgar score 4-6 were 2 and apgar score more than 7 were 97 among the decreased fetal movements group while all cases had apgar score more than 7 among good fetal movements group. In our study 5 babies were admitted for birth asphyxia, 5 babies for meconium aspiration syndrome, 3 babies for low birth weight and 3 babies for large for gestational age among the decreased fetal movements group. Seven babies were admitted to nursery for observation due to large for gestational age ( to rule out neonatal hypoglycemia) among cases with good fetal movements. There was only $1 \%$ early neonatal death among cases with decreased fetal movements (all due to severe birth asphyxia, meconium aspiration syndrome and severe IUGR). There was no perinatal mortality among cases with good fetal movements.

\section{CONCLUSION}

Fetal movement is a sign of fetal well-being. Decreased fetal movement is a common cause for visit to antenatal clinic or admission room. It is associated with antepartum fetal distress, oligohydramnios, polyhydramnios, meconium stained liquor during labor, increased rate of instrumental and Cesarean delivery and various neonatal morbidities and mortalities.

\section{REFERENCES}

1. OlesenAnette G, Svare Jens A. Decreased fetal movements: background, assessment, and clinical management. Acta Obstet Gynecol Scand. 2004; 83:818-26.

2. Sadovskey E, Ohel G, Havazeleth H, Steinwell A, Penchas S. The definition and the significance of decreased fetal movements. ActaobstetGynecol Scand. 1983; 62:409-13.

3. Tveit JVH, Saastad Eli ,Stray-Pedersen B, Bordahi Per E, Flenady V, Fretts R.et al. Reduction of late stillbirth with the introduction of fetal movement information and guidelines-a clinical quality improvement. BMC Pregnancy Childbirth. 2009;9:32.

4. Heazell AEP, Green M, Wright C, Flenady V, Froen F. Midwives' and obstetricians' knowledge and man agement of women 
presenting with decreased fetal movements. ActaObstetriciaet Gynecological. 2008;87:331-39.

5. Haws RA, Yakoob MYY, Soomro T, Menez EV, Darmstadt GL et al. Reducing stillbirths: screening and monitoring during pregnancy and labor. BMC Pregnancy and Childbirth. 2009;9(Suppl1):S5.

6. Solt I, Divon MY. Fetal surveillance tests. In: Blazer S, Zimmer EZ, editor. The Embryo: Scientific Discovery and Medical Ethics. Basel: Karger. 2005;pp.291-308.

7. Flynn AM, Kelly J, Mansfield H, Needham P, O'Conor M, Viegas O. A randomized controlled trial of non-stress antepartum cardiotocography. Br J ObstetGynaecol. 1982;89:427-33.

8. Rayburn W, Greene J, Donaldson M. Nonstress testing and perinatal outcome. J Reprod Med. 1980;24:191-96.

9. Evertson LR, Gauthier RJ, Collea JV. Fetal demise following negative contraction stress tests. Obstet Gynecol. 1978;51:671-73.

10. Rayburn W, Zuspan F, Motley ME, Donaldson M. An alternative to antepartum FHR testing. Am JObstet Gynecol. 1980;138:223-26.

11. Rabinowitz R, Persitz E, Sadovsky E. The relation between fetal heart accelerations and fetal movements. Obstet Gynecol. 1983;61:16-28.

12. Lee CY, Drukker B. The no stress test for antepartum assessment of fetal reserve. Am J Obstet Gynecol. 1979;134:460-70.

13. Valentin L, Marsal K. Pregnancy outcome in women perceiving decreased fetal movement. Eur J Obstet GynecolReprod Biol. 1987;24:23-32.

14. Heazell AEP, Sumathi GM, Bhatti NR. What investigation is appropriate following maternal perception of reduced fetal movements? Journal of Obstetrics and Gynecology. 2005;25(7):648-50.

15. Whitty JE, Garfinkel DA, Divon MY. Maternal Perception of decreased fetal movement as an indication for antepartum testing in a low risk population. Am J Obstet Gynecol. 1991;165:1084-88.

16. Sherer DM, Spong CY, GhidiniA,Minior RK, Salafia CM. Decreased amniotic fluid Volume at $<32$ weeks of gestation is associated with decreased fetal movements. Am J Perinatol. 1996;13:479-82.

17. Locatelli A, Zagarella A, Toso L, Assi F, Ghidini A, Biffi A. Serial assessment of amniotic fluid index in uncomplicated term pregnancies: prognostic value of amniotic fluid reduction. J Matern Fetal Neonatal Med. 2004;15:233-36.

18. Baron C, Morgan MA, Garite TJ. The impact of amniotic fluid volume assessed intrapartum on perinatal outcome. Am J Obstet Gynecol. 1995;173:167-74.

19. Sinha D, Sharma A, Nallaswamy V, JayagopalN ,Bhatti N. Obstetric outcome in women complaining of reduced fetal movements. Journal of Obstetrics and Gynecology. 2007;27(1):41- 43. 